\title{
High sensitivity liquid analyte filled photonic crystal fiber refractive index sensor covered by symmetrical metalnano-films based on surface plasmon resonance
}

\section{Zhenkai Fan ( $\square$ zhkfan@gmail.com )}

Hebei University of Science and Technology https://orcid.org/0000-0003-3365-6674

Jianye Qin

hebei keji daxue: Hebei University of Science and Technology

Guochen An

hebei keji daxue: Hebei University of Science and Technology

Yuqiu Fan

hebei keji daxue: Hebei University of Science and Technology

Yinping Zhang

hebei keji daxue: Hebei University of Science and Technology

\section{Xiuqing Zhang}

hebei keji daxue: Hebei University of Science and Technology

Haishan Liu

hebei keji daxue: Hebei University of Science and Technology

Shichao Chu

Hebei University of Science and Technology

\section{Research Article}

Keywords: Sensor, Surface plasmon resonance, Photonic crystal fiber

Posted Date: February 24th, 2022

DOI: https://doi.org/10.21203/rs.3.rs-1232533/v1

License: (c) (1) This work is licensed under a Creative Commons Attribution 4.0 International License. Read Full License 


\title{
High sensitivity liquid analyte filled photonic crystal fiber refractive index sensor covered by symmetrical metalnano-films based on surface plasmon resonance
}

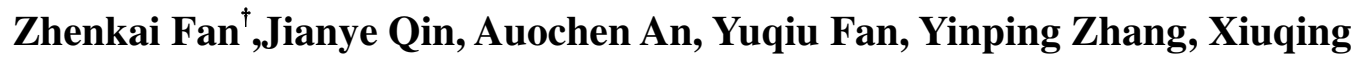 \\ Zhang, Haishan Liu,and Shichao $\mathrm{Chu}^{\ddagger}$ \\ School of Information Science and Engineering, Hebei University of Science and \\ Technology, Shijiazhuang 050018, China.
}

Abstract A high sensitivity of photonic crystal fiber (PCF) with a tunableliquid core refractive index sensor based on surface plasmon resonance (SPR) induced bythe symmetric metal films is proposed in this paper. The SPR excitation materials are chose as gold and silver. The transmission characteristics and sensing performance of the SPR-PCF sensor with different geometrical parameters were analyzed by using finite element method (FEM) and Maxwell equation. The final results show that when thediameter of the central hole is $1.6 \mu \mathrm{m}$ and the thickness of the gold film is $40 \mathrm{~nm}$, the SPR occurs at the wavelength of $1400 \mathrm{~nm}$, and the confinement loss can reach $17398 \mathrm{~dB} / \mathrm{m}$. The average sensitivity of the SPR-PCFsensor can reach $7200 \mathrm{~nm} / \mathrm{RIU}$, and the linear fitting constant is $\mathrm{R}^{2}=0.99959$, which is expected to have important applications in the direction of liquid refractive index detection.

Key words:Sensor; Surface plasmon resonance; Photonic crystal fiber

Corresponding author.

${ }^{\dagger}$ Email address: zhkfan@hebust.edu.cn

${ }^{\ddagger}$ Email address: 1241061647@qq.com

\section{Introduction}

PCFs are generally composed of fiber core, air holes and cladding, which are also called microstructure fibers(MFs). Compared with traditional fiber, it has special properties such as endlesslysingle-mode operation, extraordinary chromatic dispersion, high birefringence and high or low nonlinearity. At present, PCFs have been widely developed in the design of optical fiber devices because of their more flexible and controllable properties and the diversity of prepared materials. By filling liquid ${ }^{[1]}$, liquid crystal ${ }^{[2]}$, semiconductor ${ }^{[3]}$ or metal coating ${ }^{[4-5]}$ into the air hole of the fiber core or cladding, the characteristics of the PCF can be changed, and the application 
fields of PCF can be enriched.At present, its applications are mainly concentrated in the fields of high sensitivity sensing ${ }^{[6-8]}$, polarization-dependent nonlinearity and terahertz band optical transmission.

The metal film is coated or filled in the air holes of PCF, which can obtain properties that ordinary fiber does not have. This has become a hotspot for people to study PCF ${ }^{[9-10]}$. SPR can be excited by coating or filling gold film in the air hole of PCF, which is the result of light acting on metal surfaces. Total internal reflection occurs when light travels from a denser medium to a less dense medium and the angle of incidence is greater than critical angle. The completely reflected light wave does not disappear completely, but forms a vanishing wave. When total internal reflection occurs, the TM wave in the fading wave of incident light will interact with the metal film. It is exciting the free electrons oscillations along the surface of the metal laye in the direction of the PCF. Thus, surface plasma waves (SPW) are formed, and this phenomenon is called $\mathrm{SPR}^{[11]}$. Applying SPR to PCF as sensor has become a novel research topic.

In recent years, the SPR-PCFsensor has been developed rapidly. It has attracted more and more experts' attention in the field of optical fiber sensing. In 2012, Yang P. et al. proposed a selectively filled PCF temperature sensor, in which a liquid with a large thermal optical coefficient was filled on the second hole, and then a gold film was selectively coated on some other air holes, resulting in a final temperature sensitivity of $720 \mathrm{PM} /{ }^{\circ} \mathrm{C}^{[12]}$. In 2014, An G. et al. designed a rectangular lattice D-type PCF refractive index sensor based on SPR and deposited gold film on the plane, which is greatly simplifying the production process. Moreover, the average sensitivity could reach $8129 \mathrm{~nm} / \mathrm{RIU}^{[13]}$ within the refractive index range of 1.35-1.41. In 2015, Ahmmed A. R. proposed a PCF sensor based on graphene-silver. A thin layer of Graphene coated on the surface of silver can prevent its oxidation. The average sensitivity of the sensor can reach $3000 \mathrm{~nm} / \mathrm{RIU}^{[14]}$. In 2019, Fan Z. proposed a wide range and high sensitivity SPR-PCF sensor, and the transmission performance of the sensor was numerically analyzed and calculated ${ }^{[15]}$. In our structure, two symmetrical gold film are coated on the vertical axis of air holes of the PCF, and then the influence of the PCF on the sensing performance of the sensor is analyzed by changing other 
structural parameters of the PCF.Finally, the sensor proposed by us is expected to be applied to various aspects such as medical detection ${ }^{[16]}$, chemical detection ${ }^{[17]}$, and biological sensing ${ }^{[18]}$.

In this paper, A high sensitivity SPR-PCF refractive index sensor based on center large liquid core and metal film covering is proposed, Maxwell equation is calculated by FEM and the transmission performance of the sensor is analyzed and discussed. The metal films are selectively filled in the air holes of the second layer of PCF, and liquid are infiltrated into the air holes of the covered metal film and the center hole. By changing the type of metal film and the structure parameters of PCF, the sensing performance of the SPR-PCF sensor is optimized. Finally, the simulation results show that the sensitivity curve follows the linear fitting rule in the measurement range of refractive index. The average sensitivity of the SPR-PCF sensor can reach 7200 $\mathrm{nm} / \mathrm{RIU}$, and the high linearity is $\mathrm{R}^{2}=0.9959$, which is expected to obtain important applications in the field of optical fiber sensing and refractive index detection.

\section{Design and theory}

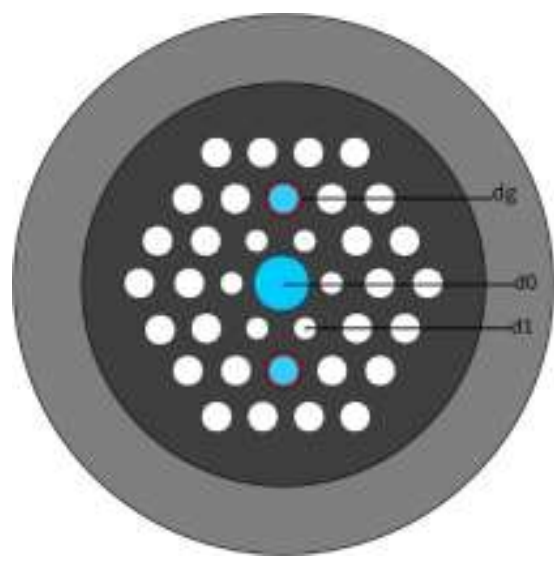

(a)

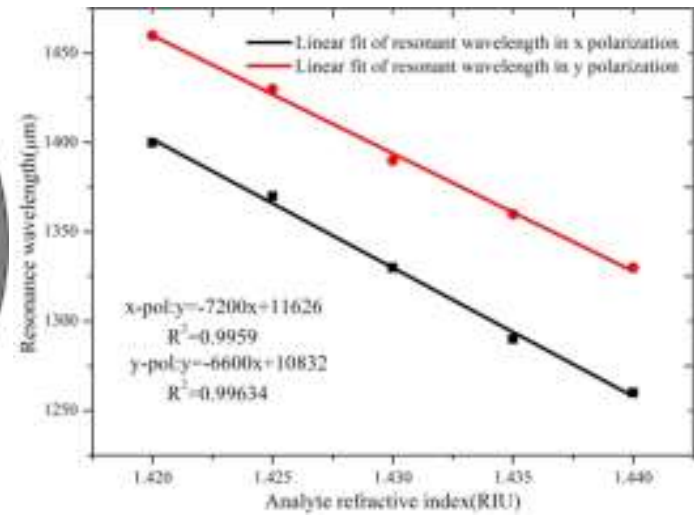

(b)

Figure 1. (a) The cross-section of the designed SPR-PCF sensor, (b) the finite element mesh and scattering boundary condition in the calculation process

The cross-section of the high-sensitivity SPR-PCF sensor is shown in Figure. 1(a).First of all, the cladding air holes arranged in triangular lattice, and the pitch size is $\Lambda=2 \mu \mathrm{m}$. The diameter of the center large hole is $1.6 \mu \mathrm{m}$, which is filled with liquid analytes used to tune the effective refractive index of the fiber core surrounded by a layer of small air hole with the diameter of $0.8 \mu \mathrm{m}$. As the two air holes in the second 
layer are coated with a gold film, the presence of small holes can increase the intensity of SPR, andthe thickness of the gold film is $t=40 \mathrm{~nm}$. The two air holes coated with the gold film are also filled with liquid analytes, which can improve the sensitivity of SPR to the liquid refractive index.The radius of ordinary air holes is 0.5 $\mu \mathrm{m}$. The refractive index of the liquid analyte varies from 1.420 to 1.440 , and the refractive index of air is 1 . The pure silicon is selected as the background material of the PCF.

Figure.1 (b) shows the linear fitting curve of PCF structurewitch is optimized. We chose gold as the coating metal, and the diameter of thecore hole is $1.6 \mu \mathrm{m}$. The refractive index sensitivity of PCF in $\mathrm{x}$ - and y-polarization reached 7200 and 6600nm/RIU,respectively, and the linear fitting constants were 0.9959 and 0.99634 , respectively. It achieves excellent sensitivity and ultra-high linearity in $\mathrm{x}$ - and y-polarization.Pure silica is selected as the background material for PCF. Its dispersion is expressed by the Sellmeier equation:

$$
\mathrm{n}(\lambda)=\sqrt{1+\frac{\mathrm{B}_{1}^{2}}{\lambda^{2}-\mathrm{C}_{1}}+\frac{\mathrm{B}_{2}{ }^{2}}{\lambda^{2}-\mathrm{C}_{2}}+\frac{\mathrm{B}_{3}{ }^{2}}{\lambda^{2}-\mathrm{C}_{3}}}
$$

where $\lambda$ represents the wavelength of free space, and other fitting constants are referred in Ref.[20]. Generally, in the PCF structure, the gold, silver, aluminum, copper, etc. can be selected as the coating or filling metal film material. In this paper, gold is selected as the material of the metal film. The dielectric constant of gold is defined by the Drude-Lorentz equation:

$$
\varepsilon_{\text {gold }}=\varepsilon_{\infty}-\frac{\omega_{\mathrm{D}}{ }^{2}}{\omega\left(\omega+\mathrm{j} \gamma_{\mathrm{D}}\right)}-\frac{\Delta \varepsilon \cdot \Omega_{\mathrm{L}}{ }^{2}}{\left(\omega^{2}-\Omega\right)-\mathrm{j} \Gamma_{\mathrm{L}} \omega}
$$

$\varepsilon_{\text {gold }}$ is the relative permittivity of gold. Other parameters are introduced in the Ref.[21], and we can use them directly. When the dielectric constant of the metal and the transmission constant of the PCF meet the phase matching condition, the light energy of the fiber core is transmitted to the metal surface to form a surface plasmon polariton (SPP) mode, and the resonance coupling for the core mode and the SPP moderesults in the SPR phenomenon.In recent years, the SPR effect has been widely used in PCF polarization filters [22], biosensors, polarization beam splitters[23] etc., 
and it plays an important role in the development and research of PCF devices.

\section{Results and analysis}

In this paper, FEM [24] and Maxwell's equation are used to analyze and discuss the transmission performance of SPR-PCF sensor. The refractive index of the analyte liquid is in the range of $1.420-1.425$ with a step of 0.005 .Figure. 2 shows the refractive index of the SPP mode and fundamental mode dependance on operation wavelength when the analyte refractive index is 1.420 for $\mathrm{x}$ and y-polarization. According to Figure. 2(a), It can be clearly seen that the refractive index of the PCF mode decreases with the increase of incident wavelength in x-polarization. The effective refractive index curve of the fundamental mode of the PCF suddenly appears an obvious jumping wavelength of $1400 \mathrm{~nm}$, where theconfinement loss reach to a peak.According to Figure. 2(b), we can find that the y-polarization is similar to the x-polarization, but the SPR of the PCF in the y-polarization occurs at a wavelength of $1460 \mathrm{~nm}$. The SPR is generated because the core mode of PCF meets the phase matching condition with the second-order SPP mode at the resonant wavelength, and the energy of the core mode of the PCF is transferred to the metal surface. The SPR is very sensitive with the change of the refractive index of the liquid analyte, so we can get the refractive index of the liquid analyte by detecting the change of resonance wavelength and resonance intensity.
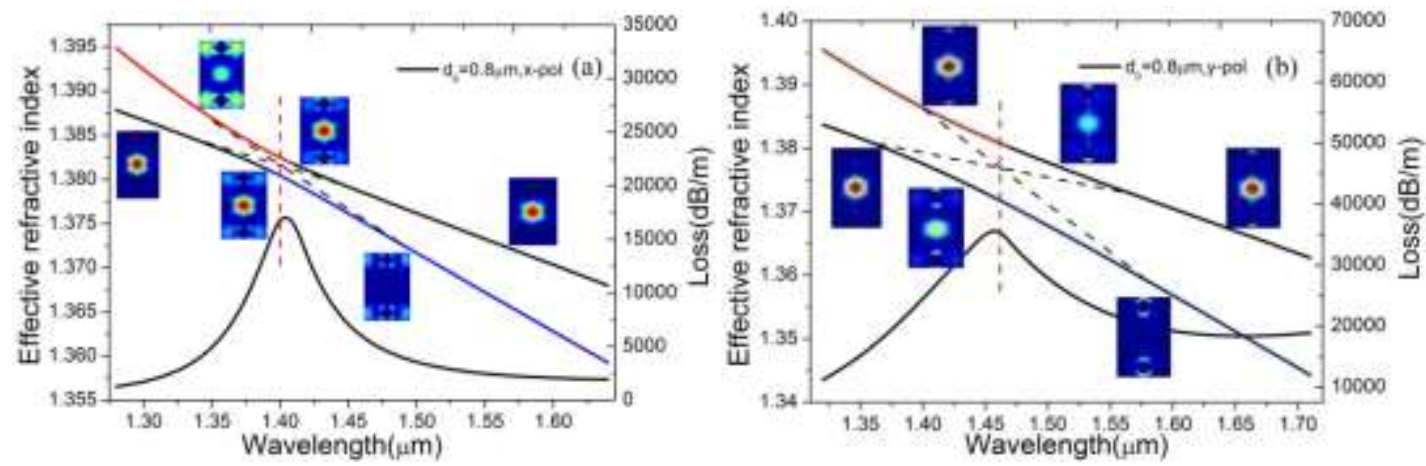

Figure 2. The loss and refractive index of the PCF dependance on the operation wavelength when the analyte refractive index na is 1.420 for (a) $x$ - and (b) $y$ - polarization.

Figure3(a) and (b) show the dependence of the effective refractive index of the $x$ and y-polarization directions on the wavelength when the radius $d_{0}$ is $0.5 \mu \mathrm{m}, 0.6 \mu \mathrm{m}$, 
$0.7 \mu \mathrm{m}$, and $0.8 \mu \mathrm{m}$, respectively. It can be clearly seen from Figure. 3(a) that the effective refractive index decreases as the incident wavelength increases in the x-polarization direction. In addition, each effective refractive index curve has a jumping point at the resonance wavelength, where the SPR has occurred at this time. Furthermore, the effective refractive index decreases regularly as the radius $\mathrm{d}_{0}$ increases. Figure. 3(b) shows the dependence of the effective refractive index of the $y$-polarization directions on the wavelength when the radius $d_{0}$ is $0.5 \mu \mathrm{m}, 0.6 \mu \mathrm{m}$, $0.7 \mu \mathrm{m}$, and $0.8 \mu \mathrm{m}$, respectively. From the Figure. 3(b), we can clearly see that the relationship between the effective refractive index of the PCF and the incident wavelength is similar for the $\mathrm{x}$ and $\mathrm{y}$-polarization directions.
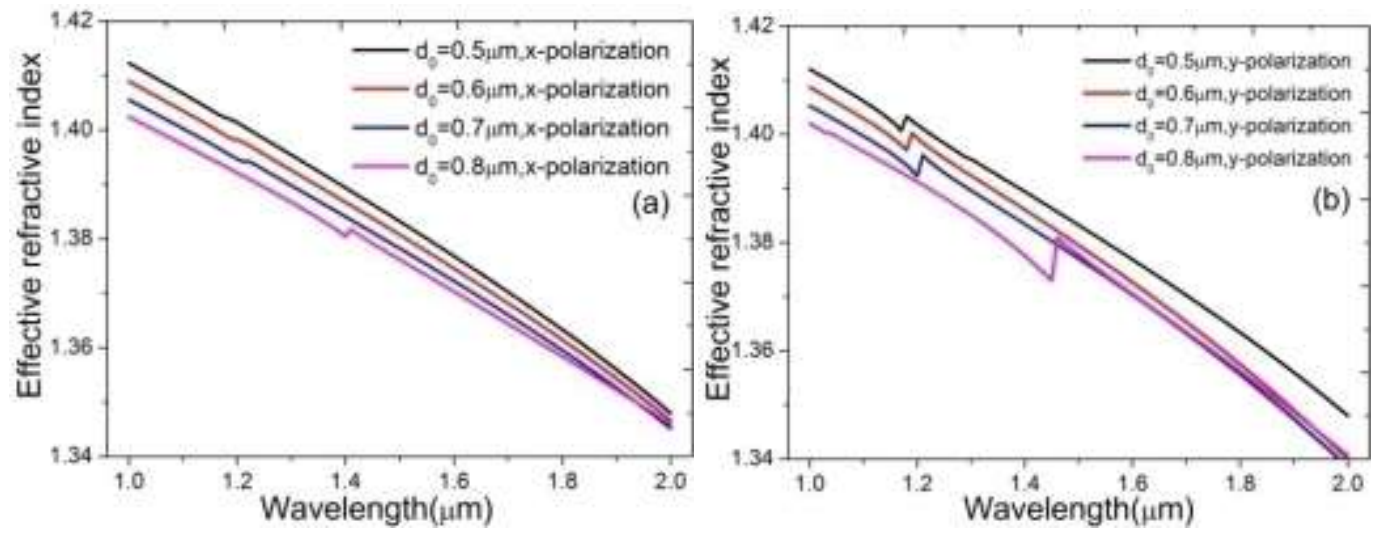

Figure 3 the dependences of the effective refractive indexes of the (a) $x$ and (b) y-polarization on the wavelength when the radius $\mathrm{d}_{0}$ is $0.5 \mu \mathrm{m}, 0.6 \mu \mathrm{m}, 0.7 \mu \mathrm{m}$, and $0.8 \mu \mathrm{m}$, respectively.

The confinement loss is an important parameter for the transmission performance of the SPR-PCF sensor. However, the strength of the confinement loss must be different for filling the different metal in the holes of the PCF. Figure4 shows the dependence of confinement loss of this PCF on operation wavelength when theradius of airhole $d_{0}$ is $0.5 \mu \mathrm{m}$ and $0.6 \mu \mathrm{m}$, respectively and the refractive index of the liquid analyte varies between 1.420 and 1.440.Figure. 4(a) and(b) represent the dependence of confinement loss on operation wavelength when the air holes of PCF are coated with goldfilm in the x-polarization direction. Figure. 4(c) and (d) represent the dependence of confinement loss on wavelength forfilling the PCF with silver film in the x-polarization direction.

It is clear from Figure. 4(a) that when the central pore $\mathrm{d}_{0}=0.5 \mu \mathrm{m}$, the limiting loss has a peak at the corresponding resonance wavelength in the x-polarization 
direction.The loss peak is gradually moving toward the short wavelength direction as the analyte refractive index increases from 1.42 to 1.44 , which has a blue shift. The resonance wavelength has moved from the $1180 \mathrm{~nm}$ to $1150 \mathrm{~nm}$, and the intensity at the resonance wavelength is gradually reduced from the $23412.48 \mathrm{~dB} / \mathrm{m}$ to 12550.74 $\mathrm{dB} / \mathrm{m}$. As shown in Figure. 4(c), it can be found that when the center air hole $\mathrm{d}_{0}=0.5 \mu \mathrm{m}$, the resonance wavelength blue shifts as the refractive index of the analyte increases.The resonance wavelength moves from the $1200 \mathrm{~nm}$ to $1170 \mathrm{~nm}$, and the loss peak value gradually decreases.Furthermore, we can clearly see in Figure 4 that in the x-polarization direction, all the resonance wavelengthsblue shift as the refractive index of the liquid analyte increases.The blue shift is most obvious in Figure $4(\mathrm{~b})$, when the center air hole $\mathrm{d}_{0}$ is $0.6 \mu \mathrm{m}$ and the filling metal is chose as gold. The resonance wavelength has moved from the $1200 \mathrm{~nm}$ to $1150 \mathrm{~nm}$, and the peak of the confinement loss has changed from the $30722.57 \mathrm{~dB} / \mathrm{m}$ to $13170.35 \mathrm{~dB} / \mathrm{m}$.It can be found that the resonance wavelength and confinement loss of the PCF with the center air hole $\mathrm{d}_{0}=0.6 \mu \mathrm{m}$ are very sensitive to the change of the refractive index of liquid analyte.
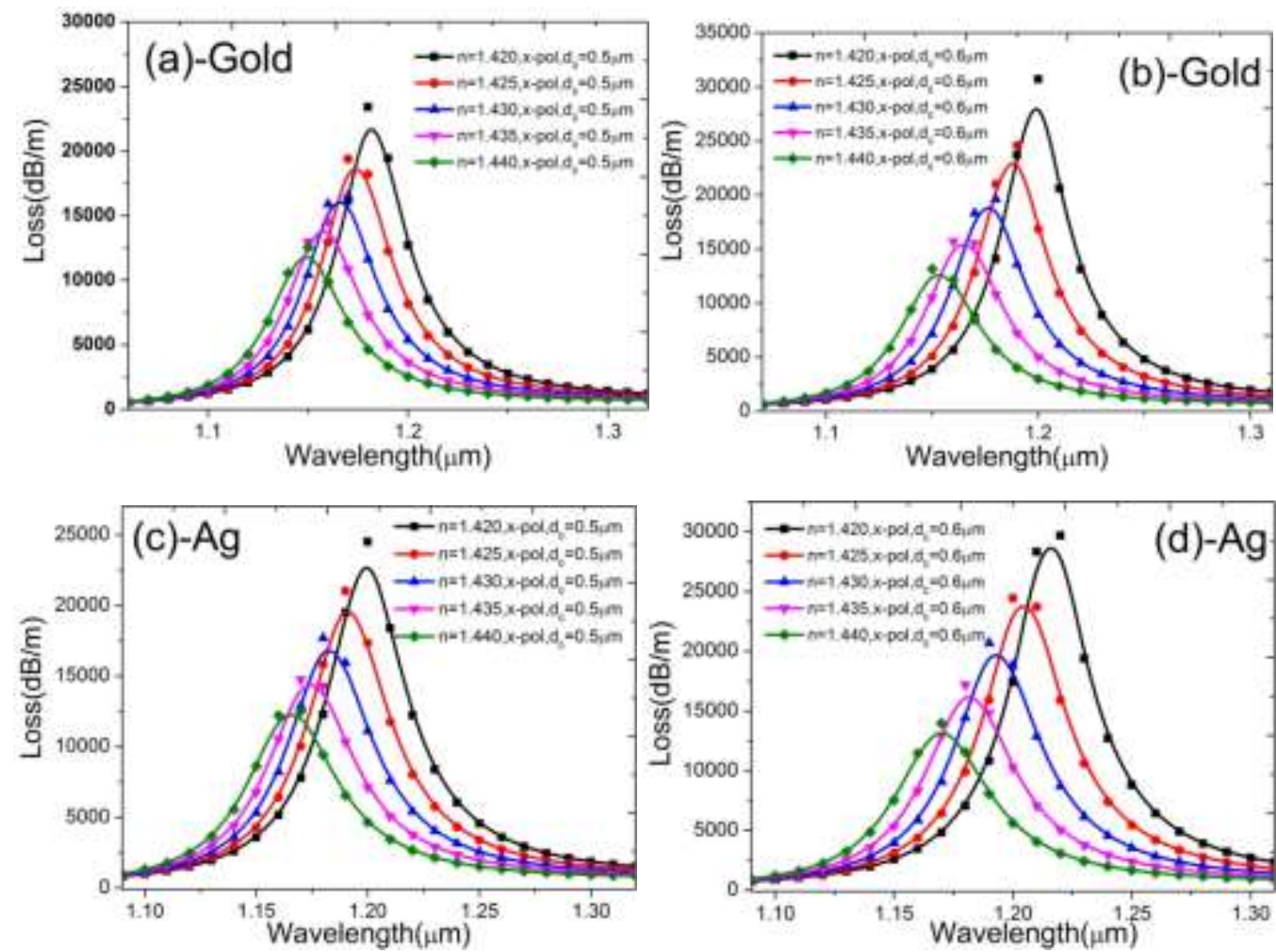

Figure 4. the dependence of confinement loss of PCF on wavelength for the radius of $\mathrm{d}_{0}$ is $0.5 \mu \mathrm{m}$ and $0.6 \mu \mathrm{m}$ when the (a), (b) gold film and (c), (d) silver film are filled PCF respectively. 
Figure 5. shows the dependence of confinement loss of this PCF on wavelength when the hole $\mathrm{d}_{0}$ is $0.5 \mu \mathrm{m}$ and $0.6 \mu \mathrm{mrespectively,and} \mathrm{the} \mathrm{refractive} \mathrm{index} \mathrm{ofliquid}$ analyte varies from 1.420 to1.440.Figure.5(a) and (b) represent the dependence of confinement loss on wavelength of the PCF filled with gold film in the y-polarization direction. It's similar to the x-polarization direction, according to Figure. 5(a), we can clearly see that in the y-polarization direction, and the confinement loss has a peak at the corresponding resonance wavelength. The resonant wavelength shifts toward the shorter wavelength as the refractive index of the liquid analyte increases. The resonance wavelength has moved from $1170 \mathrm{~nm}$ to $1140 \mathrm{~nm}$, and the peak of confinement loss is also reduced from $97802.84 \mathrm{~dB} / \mathrm{m}$ to $86316.94 \mathrm{~dB} / \mathrm{m}$. Figure. 5 (c) and (d) represent the dependence of confinement loss on wavelength of the PCF filled with gold film in the y-polarization direction. According to Figure. 5(c), it can be found that in the y-polarization direction, the resonance wavelength blue shifts as the refractive index of the analyte increases, and the resonance wavelength shifts from $1190 \mathrm{~nm}$ to $1150 \mathrm{~nm}$.Further,according to the four pictures in Figure 5, it can be clearly seen that in the y polarization direction, all resonance wavelengths are blue-shifted as the refractive index of the analyte increases. The blue shift is most obvious in Figure.5(b), when the centerhole $\mathrm{d}_{0}$ is $0.6 \mu \mathrm{m}$ and the filled metal material ischose as gold, and the resonance wavelength has moved from $1190 \mathrm{~nm}$ to $1140 \mathrm{~nm}$.
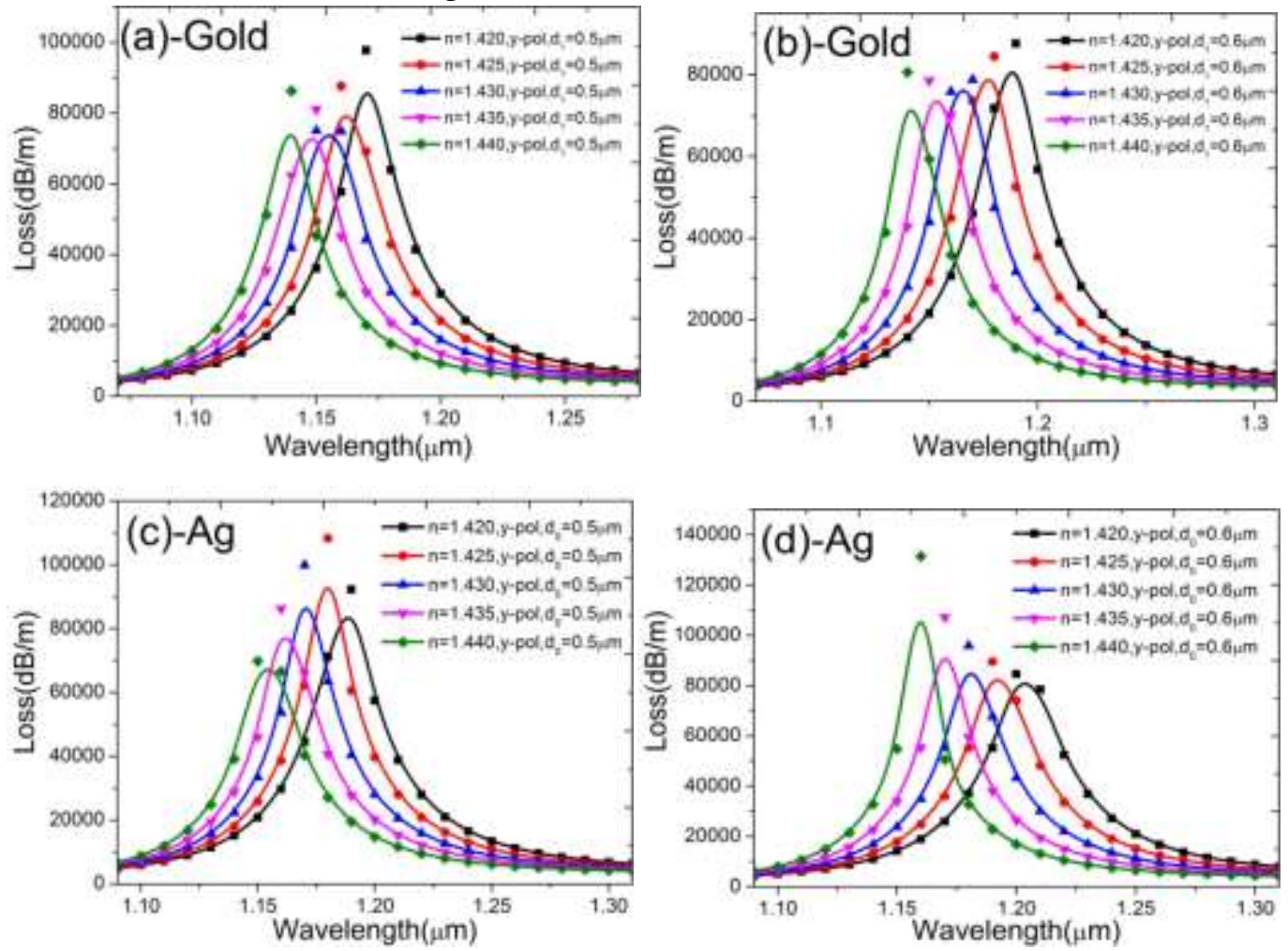

Figure 5. the dependence of confinement loss of PCF on wavelength for the radius of $d_{0}$ is 
$0.5 \mu \mathrm{m}$ and $0.6 \mu \mathrm{m}$ when the (a), (b) gold film and (c), (d) silver film arefilled PCF respectively.

Figure 6 shows the dependence of confinement loss of this PCF on wavelength when the hole $\mathrm{d}_{0}$ is $0.7 \mu \mathrm{m}$ and $0.8 \mu \mathrm{m}$, and the refractive index of the analyte varies from1.420 to1.440. Figure6(e) and (f) represent the dependence of confinement loss on wavelength of the PCF filled with gold film in the x-polarization direction. Figure 6(g) and (h) represent the dependence of confinement loss on wavelength of the PCF filled with silver film in the x-polarization direction. According to Figure 6(e), we can clearly see thatthe resonance wavelength blue shifts as the refractive index of the liquid analyte increases, the resonance wavelength has moved from $1400 \mathrm{~nm}$ to $1260 \mathrm{~nm}$, and the loss peak value has changed from $17398.32 \mathrm{~dB} / \mathrm{m}$ to $22532 \mathrm{~dB} / \mathrm{m}$.According to Figure. 6(h), We can observe that the blue shift of the resonance wavelength as the refractive index of the liquid analyte increases, the resonance wavelength has moved from $1250 \mathrm{~nm}$ to $1180 \mathrm{~nm}$, and the loss peak value has changed from $53210.81 \mathrm{~dB} / \mathrm{m}$ to $15458.26 \mathrm{~dB} / \mathrm{m}$. According to all the graphs in Figure 4 and 6 , we can know that in the $\mathrm{x}$-polarization direction, the resonant wavelengths all shifted to short wavelengths as the refractive index of the analyte increases, but the magnitude of the shift is different. In Figure. 4 and 6, the most obvious movement is shown in Figure. 6(f). At this time, the filled metal of the PCF is gold and the radius of center air hole $\mathrm{d}_{0}$ is $0.8 \mu \mathrm{m}$. This shows that the resonance wavelength is most sensitive to the change of the refractive index of the liquid analyte, and it can be used to make a high-sensitivity PCF refractive index sensor. The analysis results show that the central hole $\mathrm{d}_{0}=0.8 \mu \mathrm{m}$ is the most suitable parameter for making PCF liquid refractive index sensor. 

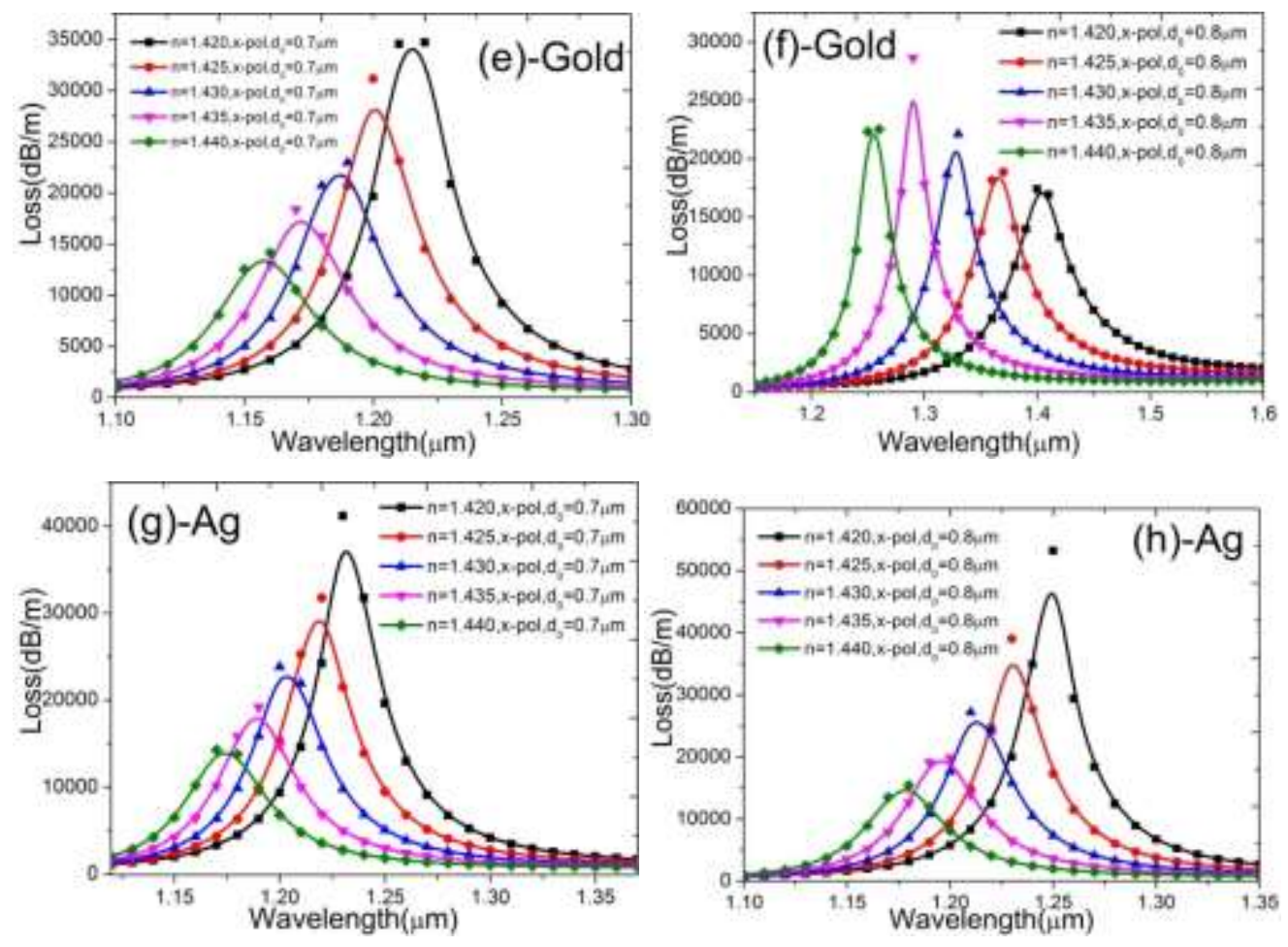

Figure6 the dependence of confinement loss of PCF on wavelength for the radius of $d_{0}$ is $0.7 \mu \mathrm{m}$ and $0.8 \mu \mathrm{m}$ when the (e), (f) gold film and (g), (h) silver film are filled PCF respectively.

Figure 7 shows the dependence of confinement loss of this PCF on wavelength when the hole $\mathrm{d}_{0}$ is $0.7 \mu \mathrm{m}$ and $0.8 \mu \mathrm{m}$, and the refractive index of the liquid analyte varies from 1.420 to1.440. Figure7(a) and (b) represent the dependence of confinement loss on wavelength of the PCF filled with gold film in the y-polarization direction. Figure. 7(c) and (d) represent the dependence of confinement loss on wavelength of the PCF filled with silver film in the y-polarization direction. According to Figure. 7(b), we can clearly see that the resonance wavelength has a blue shift as the refractive index of the liquid analyte increases, the resonance wavelength has moved from 1460nm to $1330 \mathrm{~nm}$, and the loss peak value has changed from $36051.54 \mathrm{~dB} / \mathrm{m}$ to $42758.95 \mathrm{~dB} / \mathrm{m}$. According to Figure. $7(\mathrm{~d})$, we can observe that the resonance wavelength of the PCF also has a blue shift as the refractive index of the liquid analyte increases, and the resonance wavelength has moved from $1240 \mathrm{~nm}$ to $1170 \mathrm{~nm}$. According to Figure. 5 and 7, we can know that in the y-polarization direction,the resonant wavelength shifts to short wavelengths as the refractive index of the liquid analyte increases, and the magnitude of the shift is different.From Figure. 5 and 7, the most obvious movement is Figure. 7(b). At this time, the filled metal of 
the PCF is gold and the center air hole $\mathrm{d}_{0}=0.8 \mu \mathrm{m}$, which shows that the resonance wavelength is most sensitive to the change of the refractive index of the liquid analyte.
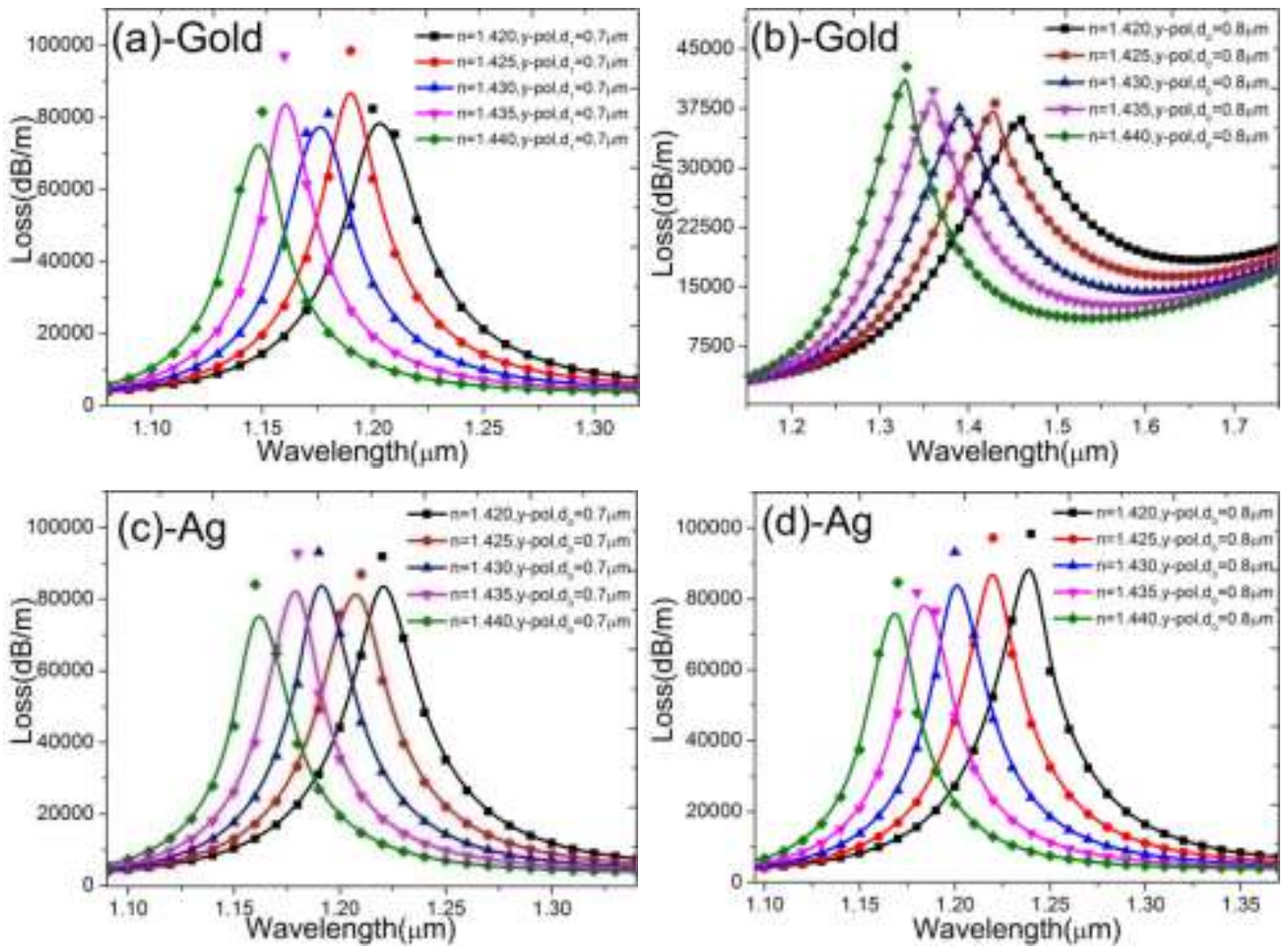

Figure 7. the dependence of confinement loss of this PCF on wavelength for the radius of $\mathrm{d}_{0}$ is $0.7 \mu \mathrm{m}$ and $0.8 \mu \mathrm{m}$ when the (a), (b) gold film and (c), (d) silver film filled PCF respectively.

It is well known that the transmission performance of PCF can be affected by coated metal on the air hole. Generally, gold, silver, aluminum and copper can be used as metal coating materials for the air holes of PCFs. Next, the sensitivity of the SPR-PCF sensor is analyzedby filling with gold and silver. Sensitivity represents the sensing performance of a sensor. The sensitivity of the SPR-PCF sensor is the gradient of the resonance wavelength with the refractive index of the liquid analyte, which is the slope of the straight line in the Figure 8. We selected multiple sets of data to calculate the average sensitivity of the SPR-PCF sensor in a given refractive index measurement range. Sensitivity can be expressed as a formula ${ }^{[25]}$ :

$$
S(\lambda)=\frac{\partial \lambda_{p e a k}}{\partial n_{a}}[n m / R I U]
$$

where $\partial \lambda_{\text {peak }}$ represents the change in the resonance wavelength corresponding to 
the loss peak of the PCF, and $\partial n_{a}$ represents the change in the refractive index of the liquid analyte.Figure. 8 shows that the fitting curve of the SPR-PCF refractive index sensor changes with the increase of the diameter of the center air hole when the refractive index of the liquid analyte increases from 1.420 to 1.440 , and the coating material of the SPR-PCF is selected as gold and silver.

As shown in Figure. 8, the average sensitivity of the SPR-PCF sensor filled with gold and silver also increases with the center air hole increases. According to Figure 8(a), the gold-coated SPR-PCF sensor has an average sensitivity of $1400 \mathrm{~nm} / \mathrm{RIUin}$ the x-polarization direction, and the silver-coated SPR-PCF sensor has an average sensitivity of $1600 \mathrm{~nm} / \mathrm{RIU}$ when the center hole $\mathrm{d}_{0}$ is $0.5 \mu \mathrm{m}$. The sensing performance of the SPR-PCF sensor is further optimized with the increase of the center air hole. Figure. 8(b) and (c) show the fitting relationship between the resonance wavelength and analyte refractive indexfor the PCF with the gold and silver film when the radius of center air hole is $0.6 \mu \mathrm{m}$ and $0.7 \mu \mathrm{m}$, respectively. When the gold films are coated in PCF with the radius of the center air hole of $0.6 \mu \mathrm{m}$ and $0.7 \mu \mathrm{m}$, and the linear fitting equations are $y=-2600 x+4894$ and $y=-3000 x+5478$ for the SPR-PCF, and the linear fitting constants are 0.97674 and 0.98246 , respectively. When the silver film is coated for the PCF with the radius of center air hole of $0.6 \mu \mathrm{m}$. The linear equation is $y=-2400 x+4624$, and the fitting constant is 0.96396 . When the radius of center air hole is $0.7 \mu \mathrm{m}$ for the $\mathrm{PCF}$, the linear equation is $\mathrm{y}=-3000 \mathrm{x}+5492$, and the fitting constant is 0.98246 . According to Figure. $8(\mathrm{~d})$, the average sensitivity of the SPR-PCF sensor filled with gold can reach $7200 \mathrm{~nm} / \mathrm{RIUwhen}$ the radius of center air hole $\mathrm{d}_{0}$ is $0.8 \mu \mathrm{m}$ for the PCF. The resonance wavelength versus theanlyte refractive index is defined as $\mathrm{y}=-7200 \mathrm{x}+11626$, and the high linearity $\mathrm{R}^{2}$ is 0.9959 . When the radius of center air hole of PCF $\mathrm{d}_{0}$ is $0.8 \mu \mathrm{m}$, the average sensitivity of the silver-filled SPR-PCF sensor can reach $3400 \mathrm{~nm} / \mathrm{RIU}$.The linear fitting equation can be expressed as $\mathrm{y}=-3400 \mathrm{x}+6076$, and the linear fitting constant is $\mathrm{R}^{2}=0.9863$. 

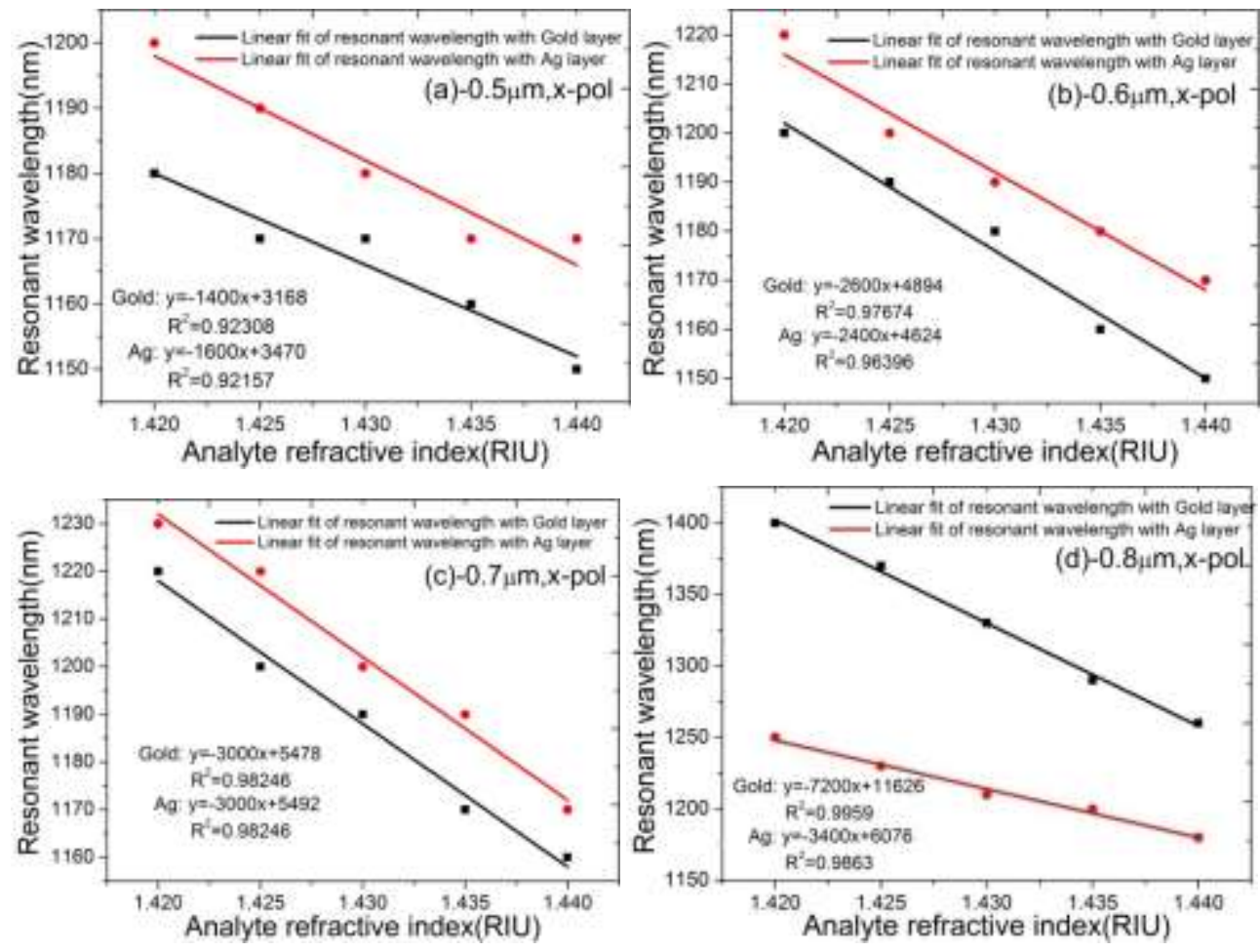

Figure 8. the resonance wavelength dependance on the liquid analyte refractive index in $\mathrm{x}$ polarization when the liquid analyte refractive index increases from 1.420 to 1.440 by a step of 0.005 , and the metal layer is chose as gold and silver.

We can clearly see in Figure 9 that when the coating materials are selected as gold and silver, the fitting curve of the SPR-PCF sensor in the y-polarization direction changes as the central pore radius increases. The average sensitivity of the SPR-PCF sensor filled with gold and silver also increases as the diameter of center air hole increases. According to Figure 9(a), the gold-coated SPR-PCF sensor has an average sensitivity of $1400 \mathrm{~nm} / \mathrm{RIUin}$ the y-polarization direction, when the radius of center air hole $\mathrm{d}_{0}$ is $0.5 \mu \mathrm{m}$. However, the silver-coated SPR refractive index sensor has an average sensitivity of $2000 \mathrm{~nm} / \mathrm{RIU}$ when theradius of center air hole $\mathrm{d}_{0}$ is $0.5 \mu \mathrm{m}$. The sensing performance of the SPR-PCF sensor is further optimized as the increase of the center air hole. From Figure 9(b) and (c), we can clearly see that when a gold film is coated in the PCF with the radius of the center air hole of $0.6 \mu \mathrm{m}$ and $0.7 \mu \mathrm{m}$. The linear equations of the SPR-PCF sensor are $y=-2600 x+4884$ and $y=-2600 x+6894$, and the linear fitting constants are 0.97674 and 0.97674 , respectively. The average sensitivity and linear fitting constant of the silver-coated SPR-PCF sensor are lower when the center air holes are $0.6 \mu \mathrm{m}$ and $0.7 \mu \mathrm{m}$. The linear fitting equations of the 
gold-coated SPR-PCF sensor are $y=-2000 x+4040$ and $y=-3000 x+5482$, respectively, and the linear fitting constants are 1 and 0.98246 when the radius of the center air hole is $0.6 \mu$ mand $0.7 \mu \mathrm{m}$.

When the center air hole $d_{0}$ is $0.8 \mu \mathrm{m}$, the average sensitivity of the gold-filled SPR-PCF sensor can reach $6600 \mathrm{~nm} / \mathrm{RIU}$, the linear fitting equation can be expressed as $y=-6600 x+10832$, and the linear fitting constant is $R^{2}=0.99634$. When theradius ofthe center air hole $\mathrm{d}_{0}$ is $0.8 \mu \mathrm{m}$, the average sensitivity of the silver-filled SPR-PCF sensor can reach $3600 \mathrm{~nm} / \mathrm{RIU}$. The linear fitting equation can be expressed as $\mathrm{y}=-3600 \mathrm{x}+6350$, and the linear fitting constant is $\mathrm{R}^{2}=0.98374$. Table 1 shows the comparison of the sensitivity and linear fitting constant of the SPR-PCF sensor filled with gold and silver under different diameters of the center air hole.It can be seen that the sensitivity and fitting constant of the gold-coated PCF are greater than those of the silver-coated PCF.In addition, the chemical stability of gold is higher than silver. Therefore, the coating metal material of the SPR-PCF sensor is selected as gold.
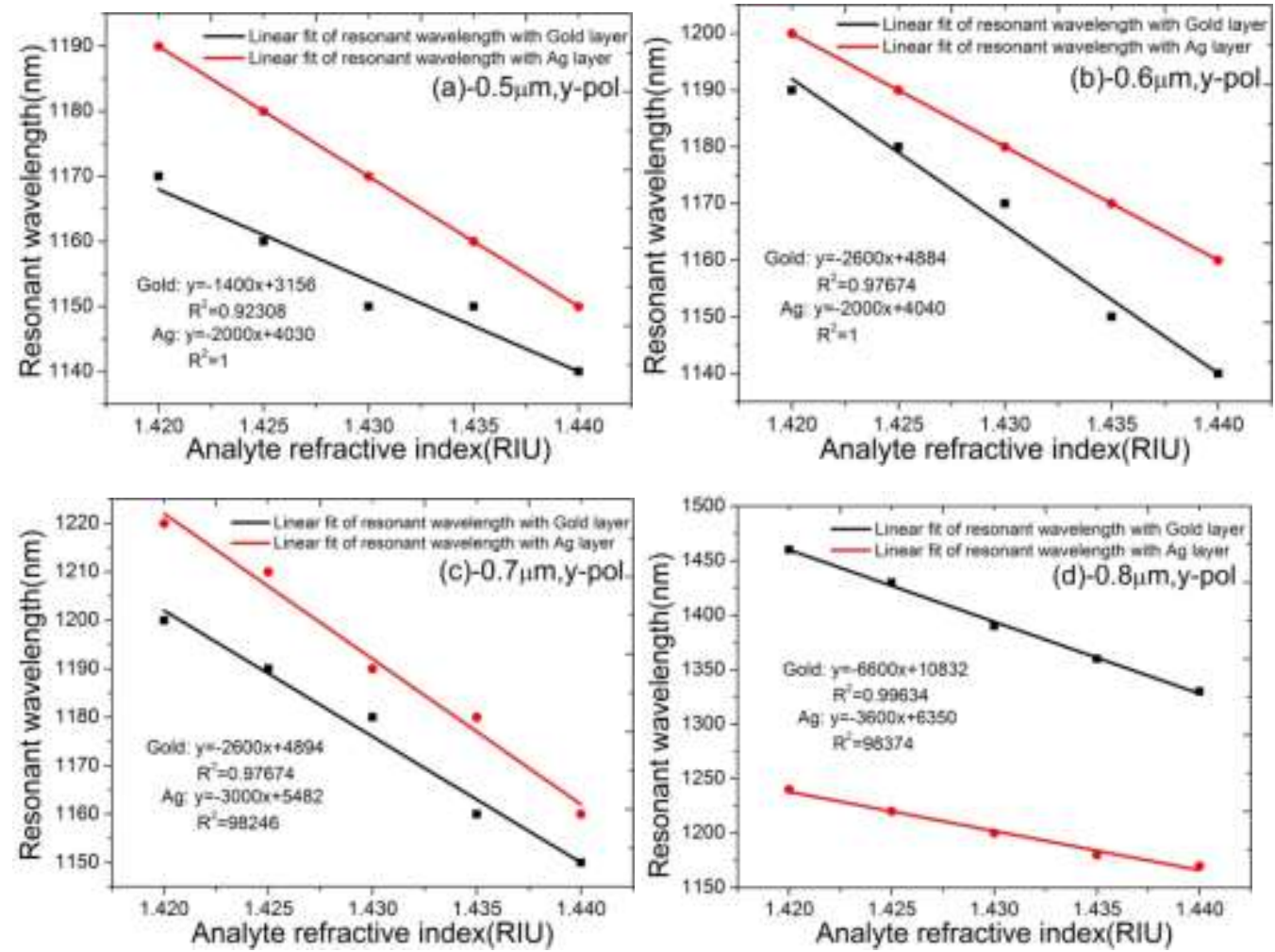

Figure 9. The resonance wavelength dependance on the liquid analyte of refractive index in the y-polarization,which increases from 1.420 to 1.440 by a step of 0.005 and the metal layer is chose as gold and silver. 
Table 1: The sensitivity and linear fitting constant of the SPR-PCF liquid refractive index sensor filled with gold and silver under different radius of the center hole.

\begin{tabular}{|c|c|c|c|c|c|c|}
\hline & Type of metal & $d_{0}(\mu m)$ & 0.5 & 0.6 & 0.7 & 0.8 \\
\hline & Gold & $S(n m / R I U)$ & -1400 & -2600 & -3000 & -7200 \\
\hline \multirow{4}{*}{$X$-pol } & \multirow{3}{*}{$A g$} & $R^{2}$ & 0.92308 & 0.97674 & 0.97674 & 0.9959 \\
\hline & & $S(n m / R I U)$ & -1600 & -2400 & -2400 & -3400 \\
\hline & & $R^{2}$ & 0.92157 & 0.96396 & 0.98246 & 0.9863 \\
\hline & \multirow[t]{2}{*}{ Gold } & $S(n m / R I U)$ & -1400 & -2600 & -2600 & -6600 \\
\hline \multirow{3}{*}{ Y-pol } & & $R^{2}$ & 0.92308 & 0.97674 & 0.97674 & 0.99634 \\
\hline & \multirow[t]{2}{*}{$A g$} & $S(n m / R I U)$ & -2000 & -2000 & -3000 & -3600 \\
\hline & & $R^{2}$ & 1 & 1 & 0.98246 & 0.98374 \\
\hline
\end{tabular}

As the incident wavelength changes, the reflection and refraction of the PCF for light will also change, and the light transmission will also change accordingly. The optical power transmittance is related to the output power and input power of the light. The light output power is calculated by the following formula ${ }^{[26]}$ :

$$
P_{\text {out }}=P_{\text {in }} \exp \left(\frac{-\alpha L}{4.343}\right)
$$

Where $P_{\text {out }}$ represents the output power of light, and $P_{i n}$ represents the input power of light. $\alpha$ represents the limiting loss of the PCF, and $L$ represents the length of the PCF. Here, we choose $1 \mathrm{~mm}$. The optical power transmittance can be calculated by the following formula ${ }^{[26]}$ :

$$
T_{r}=10 \log _{10}\left(\frac{P_{\text {out }}}{P_{\text {in }}}\right)
$$

$T_{r}$ indicates theoptical power transmittance.According to Figure 10, we can know that as the refractive index of the liquid analyte changes from 1.420 to 1.440 , the optical power transmission of the PCF filled the gold and silver all have a shift in the direction of short wavelength.The optical power transmission of the gold-filled PCF decreased from $-17 \mathrm{~dB}$ to $-22 \mathrm{~dB}$, while the optical power transmittance of the silver-filled PCF increased from $-53 \mathrm{~dB}$ to $-15 \mathrm{~dB}$. 

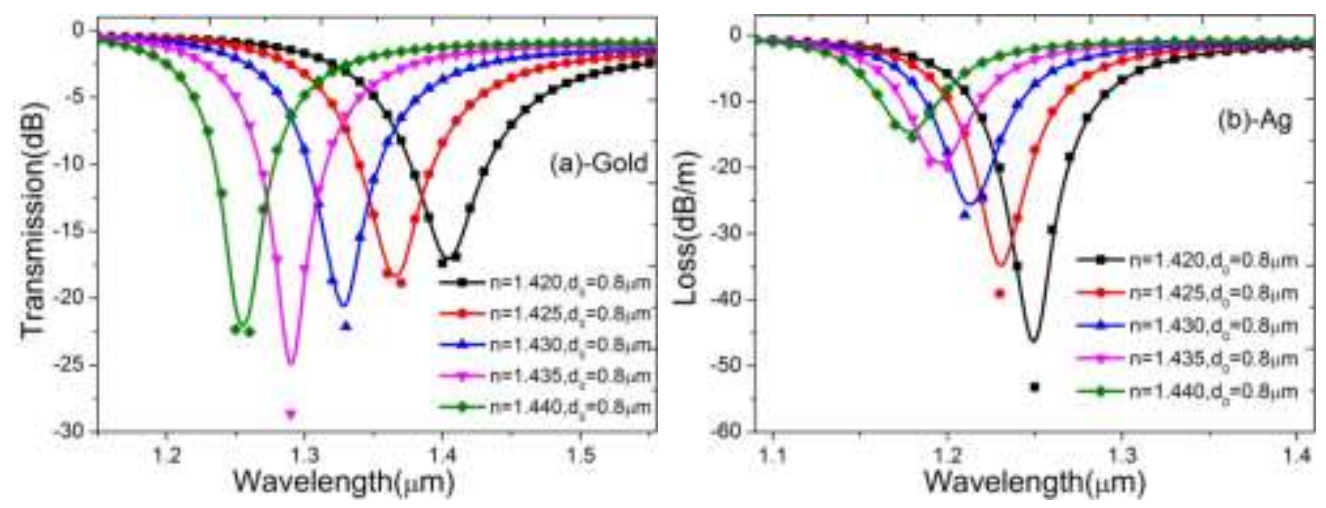

Figure 10. The optical power transmission dependence on the operation wavelength over a refractive index from 1.42 to 1.44 for (a) gold film and (b) silver film.

The figure of merit (FOM) is also an important index to measure the sensing performance of the SPR-PCF sensor. First, the FOM can be calculated with the following formula ${ }^{[15]}$ :

$$
F O M=\frac{S(\mathrm{~nm} / R I U)}{F W H M(n m)}
$$

Where S represents the average sensitivity of the SPR-PCF sensor filled with gold film and silver film, and FWHM represents the full width of the half wave of the confinement loss spectrum.According to Figure 11, we can clearly see that as the refractive index of the liquid analyte increases, the FOM of the gold-filled SPR-PCFsensor also increases steadily. The highest point is the refractive index of the liquid analyte at 1.435 , and the value is $194 \mathrm{RIU}^{-1}$.When the refractive index of the liquid analyte is 1.420 , the FOM of the SPR-PCF sensor with the gold-coated PCF is $110 \mathrm{RIU}^{-1}$.

The FOM of the silver-filled SPR-PCFsensor first increases and then decreases with the increase of the refractive index of liquid analyte. According to Figure 10, we can determine that the PCF coated with gold film is more suitable for the sensor. 


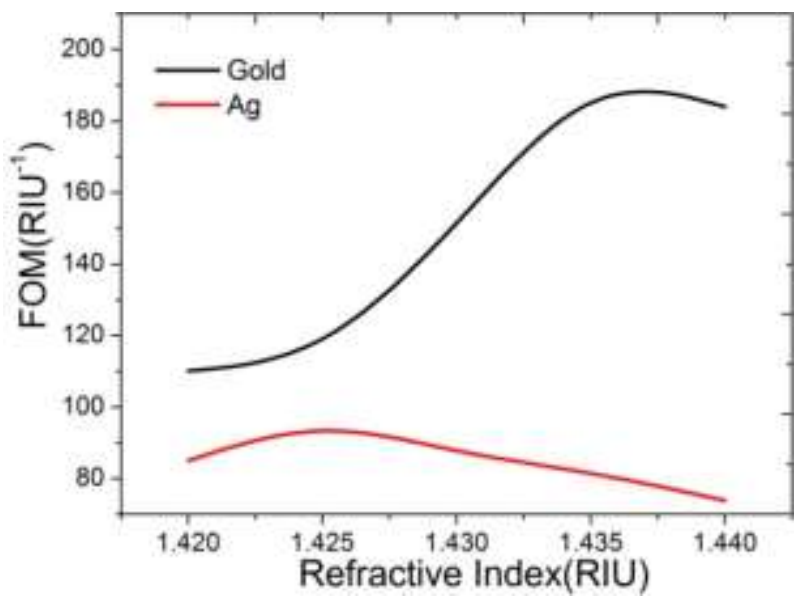

Figure11. Variation of the FOM and analyte refractive index in the SPR-PCF with gold or silver film

For the SPR-PCF sensor, the coating thickness of the metal film also has a great influence on its sensing performance. Figure 12 shows the dependence of confinement loss of this SPR-PCF on wavelength under different coating thicknesses of the gold film in SPR-PCFwhen the analyte refractive index is 1.420. Figure 12(a) and (b) represent the confinement loss dependence on the operation wavelength in $\mathrm{x}$ - and y-polarization directions, respectively.According to Figure. 12(a), it can be seen in the $\mathrm{x}$-polarization direction that the loss peak shifts to the short wavelength direction, and then shifts to the long wavelength direction when the metal film thickness is $40 \mathrm{~nm}$, and it finally moves to the short wavelength direction. The wavelength corresponding to the confinement loss peak value first decreases, then increases, and finally decreases, in other words, the resonance wavelength first blue-shifted, then red-shifted, and finally blue-shifted again. They don't show a regular movement with the increase of the thickness of gold film. However, the confinement loss peak does not increase with the increase of the metal film thickness. It can be seen from the Figure 12 (a) that the loss peak of $d_{g}=40 \mathrm{~nm}$ is smaller than the $d_{g}=20 \mathrm{~nm}, d_{g}=30 \mathrm{~nm}$, $\mathrm{d}_{\mathrm{g}}=50 \mathrm{~nm}$ and $\mathrm{d}_{\mathrm{g}}=60 \mathrm{~nm}$. Compared with Figure 12(b), it is not difficult to find that the $\mathrm{x}$ - and $\mathrm{y}$-polarization direction are similar in law.Therefore, it is known that if the metal film is too thin or thick, it is not conducive to the production of the SPR-PCF. The thickness of the metal film should be selected according to the actual situation. The optimal thickness of the gold film in this design is selected as $40 \mathrm{~nm}$. 

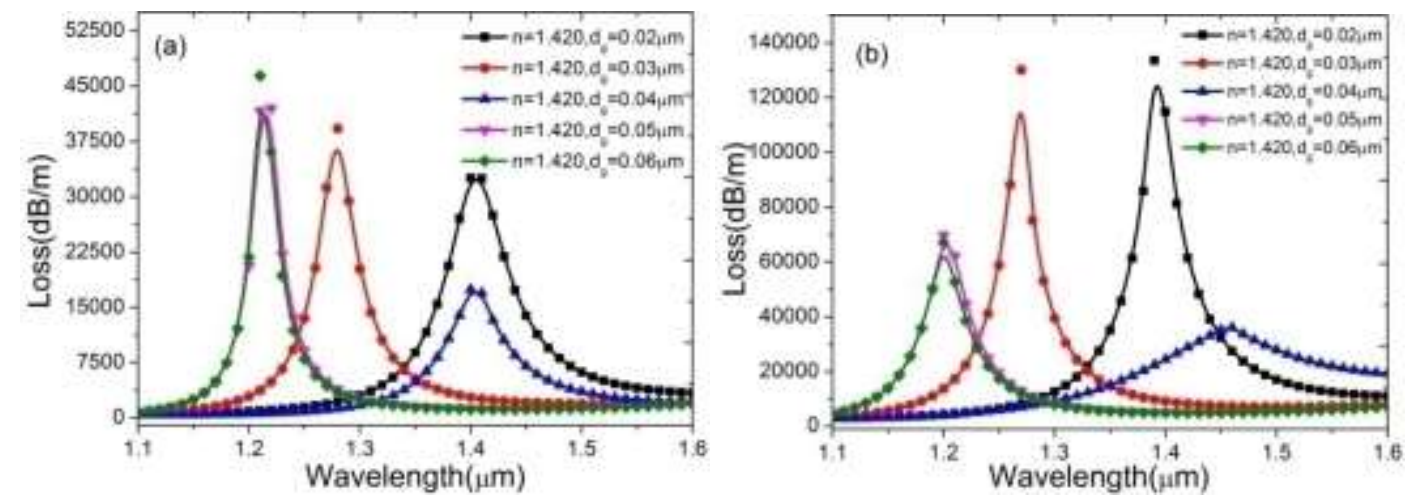

Figure 12. Variations of confinement loss with incident wavelength under different thickness of SPR-PCF coated gold film when the analyte refractive index $n_{a}$ is 1.420 for (a) $x$ - and (b) y-polarization.

\section{Conclusion}

In this paper, we designed and proposeda high sensitivity SPR-PCF refractive index sensor based ontunable liquid core and covered with metal film. We selectively coated the air holes of the SPR-PCF with a gold nano-film to stimulate the SPR. The FEM are used to analyze and discuss the sensor's sensing performance by solving the Maxwell's equation. Then the performance of the sensor is optimized by tuning the size of the center air hole and the type of filled-metal materials. The detection range of the analyte refractive index is 1.420-1.440, and the resonance wavelength was fitted linearly with the refractive index data of the analyte. Finally, it isdemonstrated that the average sensitivity of the SPR-PCF sensor can reach $7200 \mathrm{~nm} / \mathrm{RIU}$, and the linear fitting constant $\mathrm{R}^{2}$ is 0.9959 .Compared with existing sensors, the performance of the SPR-PCF sensor proposed in this paper is very competitive, and it has a very bright development prospect in the field of liquid refractive index detection.

\section{Acknowledgment}

This work was by the Natural Science Foundation of Hebei Province, China (Grant No. A2020208005), the College Young Talents Program of Hebei Province (Grant No. BJ2018040) and Hebei University of Science and Technology Talent Introduction Project(Grant No. PYB2018016).

\section{Compliance with ethical standards}

Conflict of interest: All the authors have read the manuscript and approved this for 
submission as well as no competing interests.

\section{Reference:}

1. Qian,W. W., Zhao,C. L., He,S. L., Dong,X. Y., Zhang,S. Q.,Zhang,Z. X., Jin,S. Z., Guo,J. T.,and Wei,H. F.:High-sensitivity temperature sensor based on an alcohol-filled photonic crystal fiber loop mirror. Opt. Lett. 36(9), 1548-1550(2011).

2. Hameed,M. F. O.,Balat,R. T.,Heikal,A. M., Abo-Elkhier,M. M., AboelMaaty, M. I.,and Obayya,S.S.A.:Polarization-Independent Surface Plasmon Liquid Crystal Photonic Crystal Multiplexer-Demultiplexer. IEEE Photon. J. 7(5),1-10(2015).

3. Ahmed, K., Paul,B. K.Jabin,Md. A., and Biswas,B.:FEM Analysis of Birefringence, Dispersion, and Nonlinearity of Graphene Coated Photonic Crystal Fiber. Ceramics International 45(12), 15343-15347(2019).

4. Wu,T. S., Shao,Y., Wang,Y., Cao,S. Q., Cao,W. P., Zhang, F., Liao,C. R., He,J., Huang,Y. J., Hou,M X.,and Wang, Y. P.:Surface plasmon resonance biosensor based on gold-coated side-polished hexagonal structure photonic crystal fiber. Opt. Express 25(17),20313-20322(2017).

5. Yang,X. C., Lu,Y., Liu, B. L., Xu,D. G., and Yao, J. Q.:Design of a Tunable Single-Polarization Photonic Crystal Fiber Filter with Silver-Coated, and Liquid-Filled Air Holes. IEEE Photon. J.4(9), 1-8(2017).

6. Rifat,A. A., Haider,F.,Ahmed, R., Mahdiraji,G. A., Adikan,F. R. M., andMiroshnichenko,A. E.: Highly sensitive selectively coated photonic crystal fiber-based plasmonic sensor. Opt.Lett. 43(4),891-894 (2018).

7. Lou,J. B., Cheng,T. L., and Li, S. G.:High sensitivity photonic crystal fiber sensor based on dual-core coupling with circular lattice. Opt. Fiber Technol. 48, 110-116(2019).

8. Wang Y. J., Li S. G., Li J. S., Zhang Z., Zhang S. H.,and Wu J. J.:High-sensitivity photonic crystal fiber refractive index sensor based on directional coupler.Opt. Fiber Technol. 49, 16-21(2019).

9. Wang,S. F., Chiu,M. H., Hsu,J. C., Chang, R. S., andWang, F. T.:Theoretical analysis and experimental evaluation of D-type optical fiber sensor with a thin gold film. Opt. Commun. 253,283-289(2010).

10. Liu,B. L., Lu,Y., Yang, X. C., and Yao,J. Q.:Surface plasmon resonance sensor based on photonic crystal fiber filled with gold-silica-gold multilayer nanoshells.Opt.Commun.405, 281-287(2017).

11. Liu,Q., J. Sun,D., Sun,Y. D., Ren,Z. H., Liu,C.,Lv, J. W., Wang,F. M., Wang,L. Y., Liu,W., Sun,T., and Chu, P. K.:Surface plasmon resonance sensor based on photonic crystal fiber with indium tin oxide film. Opt. Mater. 102, 109800(2020).

12. Peng,Y., Hou,J.,Huang,Z. H., and Lu, Q. S.:Temperature sensor based on surface plasmon resonance within selectively coated photonic crystal fiber.Appl. Opt. 51(26),6361-6367 (2012).

13. Liu,Q., Li,S. G., Chen,H. L., Li, J. S., and Fan,Z. K.:High-sensitivity plasmonic temperature sensor based on photonic crystal fiber coated with nanoscale gold film.Appl. Phys. Express 8(4), 046701(2015).

14. Rifat,A. A., Mahdiraji,G. A., Chow,D. M., Shee,Y. G., Ahmed,R., andAdikan, F. R. M.:Photonic Crystal Fiber-Based Surface Plasmon Resonance Sensor with Selective Analyte Channels and Graphene-Silver Deposited Core. Sensors-Basel.15(5), 11499-11510(2015).

15. Fan,Z. K.:Surface plasmon resonance refractive index sensor based on photonic crystal fiber covering nano-ring gold film. Opt. Fiber Technol. 50,194-199(2019). 
16. Islam,M. A.,Islam,M. R., A. Naser, M. A., Anzum, F., and Jaba, F. Z.: Square structured photonic crystal fber based THz sensor design for human body protein detection. J. Comput. Electron.20(1), 377-386(2021).

17. Qazi,H. H., Mohammad,A.,and Akram,M.:Recent Progress in Optical Chemical Sensors. Sensors 12(12),16522-16556(2012).

18. Hameed,M. F. O.,Alrayk,Y. K. A.,Shaalan,A. A.,Deeb,W. S. E., and Obayya,S. S. A.: Design of highly sensitive multichannel bimetallic photonic crystal fiber biosensor. J. Nanophotonics10(4),046016(2016).

19. Qin,J. Y., Meng, Z. Y., Gao,J. L., Fan,Z. K., andWang,X. J.:Surface plasmon enhanced polarization filter of high birefringence photonic crystal fiber with a partial core based on filled silver nanowire. Opt. Fiber Technol. 50,102342 (2020).

20. Wang,S., and Li,S. G.:Surface plasmon resonance sensor based on symmetrical side-polished dualcore photonic crystal fiber, Opt. Fiber Technol. 51 96-100(2019).

21. Zha,F. N., Li,J. S., Sun,P. J., and Ma,H. J.:Highly sensitive selectively coated D-shape photonic crystal fibers for surface plasmon resonancesensing, Phys. Lett. A. 383(15),1825-1830 (2019).

22. An,G. W., Li,S. G.,Yan,X., Z. Yuan,Y., andZhang,X. N.:High-birefringence photonic crystal fiber polarization filter based on surface plasmon resonance, Appl. Opt.55(6),1262-1266(2016).

23. Zhao,Y. Y.,Li,S.G. ,Wang,X. Y. ,Wang,G. Y. ,Shi,M. ,and Wu,J. J. : Design of a novel multi channel photonic crystal fiber polarization beam splitter,Opt. Commun.400,79-83(2017).

24. Meng,X. J., Li, J. S., Guo,Y., Li, S. G., Wang,Y. J., Bi,W. H., and Lu, H. B.: An optical-fiber sensor with double loss peaks based on surface plasmon resonance.Optik. 216,164938(2020).

25. An,G. W., Li,S. G., W. Qin, Zhang, W., Fan,Z. K., and Bao,Y. J.: High-Sensitivity Refractive Index Sensor Based on D-Shaped Photonic Crystal Fiber with Rectangular Lattice and Nanoscale Gold Film,Plasmonics 9(6),1355-1360(2014).

26. Guo,Z. P., Fan,Z. K., Kong,X. Z., and Meng,Z. Y.:Photonic crystal fiber based wide-range of refractive index sensor with phase matching between core mode and metal defect mode, Opt. Commun., 461,125233 (2020). 